\title{
ArcheoSciences
}

Revue d'archéométrie

$30 \mid 2006$

Varia

\section{Bednarz M., Kraese J., Reynier P., Becze-Deák J., Plateau de Bevaix 2. Histoire et préhistoire d'un paysage rural : le site des Pâquiers}

Book review

\section{Anne Gebhardt}

\section{(2) OpenEdition Journals}

Édition électronique

URL : http://journals.openedition.org/archeosciences/401

DOI : 10.4000/archeosciences.401

ISBN : 978-2-7535-1595-6

ISSN : 2104-3728

\section{Éditeur}

Presses universitaires de Rennes

\section{Édition imprimée}

Date de publication : 31 décembre 2006

Pagination : 231-232

ISBN : 978-2-7535-0456-1

ISSN : $1960-1360$

\section{Référence électronique}

Anne Gebhardt, « Bednarz M., Kraese J., Reynier P., Becze-Deák J., Plateau de Bevaix 2. Histoire et préhistoire d'un paysage rural : le site des Pâquiers », ArcheoSciences [En ligne], 30 | 2006, mis en ligne le 31 décembre 2008, consulté le 21 septembre 2020. URL : http://journals.openedition.org/ archeosciences/401; DOI : https://doi.org/10.4000/archeosciences.401 


\section{Compte rendu de lecture}

Bednarz, M., Kraese, J., Reynier, P. et Becze-Deák J., 2006. Plateau de Bevaix 2. Histoire et préhistoire d'un paysage rural : le site des Pâquiers, Neuchâtel, Service et musée cantonal d'archéologie (Archéologie neuchâteloise, 36), 2 volumes, $528 \mathrm{p}$.

Cet ouvrage en deux tomes expose les résultats d'une étude pluridisciplinaire très poussée du site des Pâquiers (plateau de Bevaix, canton de Neuchâtel, Suisse), effectuée durant trois années dans un contexte d'archéologie préventive sur 1,6 ha du tracé de l'autoroute A5.

Outre la classique étude des aménagements anthropiques liés à l'habitat, l'artisanat ou la production agricole, l'originalité de ce travail réside dans la prise en compte d'objets archéologiques isolés (perdus ou abandonnés) très dispersés sur le site, ainsi que l'étude d'éléments d'infrastructures (fossés, chemins, routes, limites parcellaires, drainage...). Au vu du contexte sédimentaire local fortement colluvionné, habituellement considéré comme difficile, voire stérile, les travaux archéologiques ont offert une large place aux sciences de la Terre et de la Vie, tout en s'appuyant sur de nombreux calages chronologiques absolus (C14, OSL, dendrochronologie, archéomagnétisme).

L'ouvrage commence par une introduction retraçant le cadre général (géomorphologie, stratigraphie) et chronologique des travaux, et consacre une large part aux différentes méthodes employées. Depuis la prospection (mécanique, géomagnétique), au traitement des données, en passant par les archéosciences (sciences de la Terre, paléotopographie, datations,...), toutes les étapes de la fouille et les principes techniques sont décrits avec un vocabulaire simple, accessible à tous et très bien illustré. Les choix méthodologiques sont d'ailleurs largement argumentés.

Confrontée aux données archéologiques, environnementales et chronologiques, c'est une approche pédologique et sédimentaire originale qui a permis d'appréhender avec précision les différentes phases colluviales et de ruissellement à l'origine de l'érosion du site et de la dispersion du matériel archéologique, pour préciser les principales phases d'occupation et d'abandon du secteur. Une vingtaine de cartes microtopographiques illustre ces différentes phases sédimentaires successives avec leurs épaisseurs et leurs axes de dépôt.

Pour chaque période, l'interaction entre les facteurs naturels et humains a été analysée en détail. Des modèles de déplacement et de mutations paysagères ont pu être propo- sés pour retracer la mise en place et les mutations du terroir. Les différentes datations obtenues (calage par le matériel, C14, OSL...) sur le site sont discutées et les étapes chronologiques, clefs de l'évolution du paysage des Pâquiers de la préhistoire à nos jours, ont permis l'élaboration d'une série de blocs diagrammes très parlants.

L'histoire des Pâquiers commence avant l'occupation humaine, par la mise en place et le modelage du substrat molassique et glaciaire, la sédimentation tardiglaciaire et la formation du sol holocène originel. La première structure anthropique datée par le radiocarbone est une trace de rubéfaction isolée et très érodée du Néolithique moyen II (Cortaillod classique), mais dont le contenu carpologique suggère un habitat. Entre le Néolithique récent et le Bronze ancien, le paysage s'est ouvert. Les analyses anthracologiques, malacologiques et micromorphologiques suggèrent la présence d'un habitat dans les environs immédiats du site au cours de l'Age du Bronze. L'Age du Fer, mal documenté en céramique, est marqué par des structures rurales (murgier, chemin creux, fossé) qui suggèrent l'agriculture. Les Pâquiers ne semblent pas habités à l'époque gallo-romaine, mais la présence d'un aqueduc et d'une carrière accrédite l'existence d'une villa à proximité du site. Le plateau de Bevaix prend un aspect plus « moderne " attesté par une succession de dispositifs de drainage plus ou moins efficaces qui marque une volonté d'aménagement foncier. Ce système cadastral orthonormé semble s'effondrer dès le BasEmpire romain. La forêt réapparaît et seuls deux fours domestiques indiquent une petite installation humaine à proximité de l'ancienne villa gallo-romaine. Au cours du vi siècle, un déboisement est attesté par deux phases de forte combustion. Au cours du haut Moyen Âge, les chemins sont successivement reconstruits et abandonnés, puis vers les $\mathrm{X}^{\mathrm{e}}$-XI $\mathrm{X}^{\mathrm{e}}$ siècles, un petit décalage angulaire $\left(2^{\circ} \mathrm{a} 10^{\circ}\right)$ affecte l'orientation des chemins. Les périodes modernes et contemporaines sont marquées par la présence de plusieurs chenaux érosifs et le creusement de nombreux drains sans doute en réaction à la forte humidité du secteur. Chemins, murgiers et alignements lithiques soulignent la persistance de la tradition gallo-romaine dans l'aménagement local du terroir. 
Cet ouvrage se termine par le bilan de cette "aventure interdisciplinaire " qui remet en cause le concept de site archéologique, et démontre que l'ensemble des sites accessibles à l'homme doivent être considérés comme un tissu archéologique, au sein duquel existe des zones de densité plus forte de vestiges (sites) reliés entre elles par une trame d'éléments plus dispersés mais toujours présents.

Voilà une monographie très complète - les spécialistes retrouveront toutes les données de terrain et d'analyses, regroupées en une série d'annexes très complètes en fin d'ouvrage -, qui montre combien la pauvreté des sites col- luvionnés n'est qu'apparente à qui veut bien se donner les moyens techniques et financiers d'une étude interdisciplinaire adéquate.

Anne GebHardt

INRAP Grand Est

UMR 6566 Civilisation Atlantique et Archéosciences,

Rennes

UMR 7011 Image et Ville, Strasbourg anne.gebhardt@wanadoo.fr 